(2) Open Access Full Text Article

ORIGINAL RESEARCH

\title{
Increased risk of hospitalization for ultrarapid metabolizers of cytochrome P450 2D6
}

This article was published in the following Dove Press journal:

Pharmacogenomics and Personalized Medicine

14 February 2017

Number of times this article has been viewed

\author{
Paul Y Takahashi' \\ Euijung Ryu ${ }^{2}$ \\ Jyotishman Pathak ${ }^{2}$ \\ Gregory D Jenkins ${ }^{2}$ \\ Anthony Batzler ${ }^{2}$ \\ Matthew A Hathcock ${ }^{2}$ \\ John Logan Black ${ }^{3}$ \\ Janet E Olson ${ }^{2}$ \\ James R Cerhan² \\ Suzette J Bielinski \\ 'Division of Primary Care Internal \\ Medicine, Department of Medicine, \\ ${ }^{2}$ Department of Health Sciences \\ Research, ${ }^{3}$ Division of Clinical \\ Biochemistry and Immunology, \\ Department of Laboratory Medicine \\ and Pathology, Mayo Clinic, Rochester, \\ MN, USA
}

Background: Cytochrome P450 2D6 (CYP2D6) is responsible for the metabolism of clinically used drugs and other environmental exposures, but it is unclear whether the CYP2D6 phenotype is associated with adverse health outcomes. The aim was to determine the association of CYP2D6 phenotype with the risk of hospitalization or an emergency department (ED) visit among a group of primary care patients.

Methods: In this study, 929 adult patients underwent CYP2D6 testing. The primary outcome was risk of hospitalization or an ED visit from January 2005 through September 2014. CYP2D6 genotypes were interpreted as 1 of 7 clinical phenotypes, from ultrarapid to poor metabolizer, and patients with the extensive metabolizer phenotype were used as the reference group. The hazard ratios (HRs) and 95\% confidence intervals (CIs) were estimated for finding the association of CYP2D6 phenotypes with the risk of hospitalization or an ED visit by using Cox proportional hazard models and adjusting for age and sex.

Results: The median age was 49 years (interquartile range, $46-52$ years); 74\% of patients had 3 or fewer chronic conditions, 285 had at least 1 hospitalization, and 496 had at least $1 \mathrm{ED}$ visit. The risk of hospitalization was higher among patients who were ultrarapid metabolizers compared to extensive metabolizers ( $47 \%$ vs $30 \%$; HR, 1.69; 95\% CI, 1.11-2.57), as was the risk of an ED visit ( $62 \%$ vs $49 \%$; HR, $1.50 ; 95 \%$ CI, 1.05-2.14). For poor metabolizers compared to extensive metabolizers, there was no difference in the risk of hospitalization (HR, $0.95 ; 95 \% \mathrm{CI}$, $0.58-1.56$ ), but there was an increase in the risk of an ED visit (HR, 1.38; 95\% CI, 0.96-1.98) (the difference was not statistically significant).

Conclusion: We found an increased risk of hospitalization or an ED visit among ultrarapid compared to extensive CYP2D6 metabolizers. Further research identifying the mechanisms of the association and ultimate clinical utility is warranted.

Keywords: cohort study, pharmacogenomics, emergency department, hospitalization

\section{Introduction}

Genomic variation within the genes controlling drug metabolism has been associated with toxicity, adverse drug reactions, and ineffective drug dosing. Furthermore, medication reactions and suboptimal therapy are the common causes of hospitalization and morbidity. For example, in a study of 106 hospitalized patients, a severe drug reaction occurred in $24 \%$ of patients. ${ }^{1}$ Cytochrome $\mathrm{P} 450$ 2D6 (CYP2DO) is of particular importance because of the number and types of medications affected. ${ }^{2}$ In addition, there is a paucity of data on the adverse health outcomes of ultrarapid or poor metabolism of CYP2D6. In a study of 149 patients admitted for major depressive disorder, poor
Correspondence: Paul Y Takahashi

Division of Primary Care Internal

Medicine, Mayo Clinic, 200 First St SW,

Rochester, MN 55905, USA

Tel +I 5072844646

Fax +l 5072660036

Email takahashi.paul@mayo.edu 
metabolizers of $C Y P 2 D 6$ were hospitalized longer than normal or ultrarapid metabolizers. ${ }^{3}$ Likewise, in a smaller study of 53 patients with depression or schizophrenia, length of stay (LOS) was longer for patients with poor metabolism than for patients with other $C Y P 2 D 6$ phenotypes. ${ }^{4}$ Furthermore, pharmacogenomic testing of CYP2D6 for risperidone was found to be cost-effective in reducing LOS. ${ }^{5}$ In case studies of children who were ultrarapid CYP2D6 metabolizers, the children had an increased risk of death. ${ }^{6}$ These case studies have led to increased warnings for codeine use with both children and breastfeeding mothers. ${ }^{7}$

Precision medicine within pharmacogenomics can be used to predict adverse health outcomes such as hospitalization. In a broad population with multiple reasons for hospitalization, the potential impact of poor or ultrarapid CYP2D6 metabolism on hospitalization and emergency department (ED) visits is not known. There are many plausible reasons to believe that $C Y P 2 D 6$ metabolism activity may indeed affect hospitalization and ED visits. For example, many commonly prescribed medications, including pain medications such as codeine and tramadol, are prodrugs activated by $C Y P 2 D 6$, and several antidepressants and antipsychotics are inactivated by $C Y P 2 D 6 .{ }^{8}$ In addition, tamoxifen, a common chemotherapeutic medication for breast cancer, is metabolized through CYP2D6 to an active moiety (endoxifen), and poor metabolizers are at an increased risk for breast cancer recurrence. ${ }^{9}$

The goal of the study was to determine the relationship between CYP2D6 phenotype and the risk of hospitalization or an ED visit. It was hypothesized that participants with extreme metabolism phenotypes (either poor or ultrarapid metabolism), compared to extensive phenotypes, would have higher rates of hospitalization or ED visits or both.

\section{Methods}

\section{Patients and setting}

This study was conducted with data from the Mayo Clinic Biobank (MCB). Specifically, the MCB enrolled over 27,000 patients and collected clinical, questionnaire, and biospecimens data from April 2009 through September 2012. ${ }^{10}$ Within the MCB, 1,013 patients were recruited for the Right Drug, Right Dose, Right Time: Using Genomic Data to Individualize Treatment (RIGHT) protocol, which sequenced 86 pharmacogenomics genes for clinical use. The protocol details have been previously reported. ${ }^{11}$ Of these patients, 84 were excluded from this study because they did not receive continuous primary care at Mayo Clinic. The 929 adult patients included in the study provided consent to the MCB for use of their data ${ }^{10}$ and afforded further written informed consent upon enrollment in the RIGHT protocol. ${ }^{11}$
The RIGHT protocol was reviewed and approved by the Mayo Clinic Institutional Review Board and the Mayo Clinic Biobank Access committee.

Demographic data, including patient age and sex, were obtained from the electronic health record (EHR). Disease burden of the patients was assessed with the use of Minnesota medical tiering assessment. ${ }^{12}$ In brief, Minnesota Health Care Home Tiering was calculated on December 31, 2010, for each patient with data from the EHR. Each outpatient or inpatient visit yielded a diagnosis containing an "International Classification of Diseases, Ninth Revision" code, which was then used to calculate the tiering score, which was based on the Johns Hopkins Adjusted Clinical Groups. ${ }^{13}$ The International Classification of Diseases, Ninth Revision codes were obtained up to 1 year in advance of tiering. Each code was then assigned to 1 of 22 major clusters for counting. Patients were tiered into 1 of 5 categories: low (no chronic conditions); basic (1-3 conditions); intermediate (4-6 conditions); extended (7-9 conditions); and complex ( $\geq 10$ conditions). Smoking status (current, former, or never smoker) and alcohol consumption data (never, once monthly or less, 2-4 times monthly, 2-5 times weekly, or $\geq 6$ times weekly) were collected from the questionnaire completed at enrollment in the MCB.

\section{CYP2D6 phenotype}

CYP2D6 genotyping was performed by using a cascade of testing as is required to obtain an accurate genotype. Genomic DNA was extracted from whole blood. Assays were performed with the xTAG CYP2D6 Kit (Luminex Corp), which incorporates multiplex polymerase chain reaction (PCR) and multiplex allele-specific primer extension. This assay uses the Luminex proprietary universal tag sorting system on the Luminex 100 xMAP platform. When necessary, a copy number assay was also completed. This assay uses real-time PCR, which interrogates 3 locations on the CYP2D6 gene and includes 1 copy number probe (FAM labeled) and 1 reference assay (VIC labeled) per reaction (Taqman Copy Number Assays Revision D, Thermo Fisher Scientific Inc.). More specifically, each copy number probe detects the genomic sequence of interest, and the reference assay serves to detect a sequence known to be present in 2 copies of a diploid genome. Relative quantitation is then used to determine the relative copy number of the target of interest in a genomic DNA sample that is normalized to 10 $\mathrm{ng} / \mu \mathrm{L}$ for each probe. Each probe is normalized to the known copy number of the reference sequence and is also compared to a calibrator sample containing known copies of the target sequence included in each run. 
Sequencing of CYP2D6 was completed in selected samples as needed in order to derive the phenotype. The resulting CYP2D6 gene was amplified by PCR. The PCR product was then purified and sequenced in both directions with fluorescent dye-terminator chemistry. Sequencing products were analyzed on an automated sequencer, and trace files were then analyzed for variations in the promoter, exons, and intron-exon boundaries for all 9 exons through use of mutation detection software and visual inspection. ${ }^{14,15}$ Finally, according to allele functionality, 7 metabolic phenotypes were reported in the EHR: ultrarapid, extensive to ultrarapid, extensive, intermediate to extensive, intermediate, poor to intermediate, and poor. The phenotype algorithms were based on Clinical Pharmacogenetics Implementation Consortium guidelines. ${ }^{16}$

The CYP2D6 genotype results were used to predict which of the 7 phenotypes was in a given sample according to the method described by Ji et al. ${ }^{16}$ Different laboratories design different prediction methods. We used current findings and thinking to develop this model, and this is used in the Mayo Clinic Department of Laboratory Medicine and Pathology. Patients who did not have a detectable gene alteration were considered to be extensive drug metabolizers (CYP2D6 $* 1 / * 1){ }^{16}$

\section{Medications}

Prescribed medications were secondary risk factors of interest for an exploratory analysis. Medications were chosen according to potential adverse events or inadequate efficacy due to poor or ultrarapid metabolism of CYP2D6. We selected medications that were used by at least $5 \%$ of the 929 patients (ie, $\geq 46$ ) according to the medication history in the EHR: oxycodone, codeine, hydrocodone, tramadol, morphine, metoprolol, and amitriptyline.

\section{Hospitalization and ED visits}

Hospitalization and ED visits were determined from EHR billing codes for inpatient stays occurring from January 1, 2005 through September 30, 2014. Outpatient surgical interventions and colonoscopies, hospitalizations for research studies, and pregnancy-related hospitalizations were excluded.

\section{Statistical analysis}

Patient demographic characteristics and CYP2D6 metabolism phenotypes were summarized with use of descriptive statistics. CYP2D6 phenotype groups were compared for demographic characteristics with use of the Pearson's chisquared tests designed for categorical variables (sex and Minnesota tiers) and the Kruskal-Wallis test for continuous variables. Patients were followed through their EHR from the date of their first clinic visit (occurring on or after January 1, 2005) until the first event of hospitalization (or an ED visit), death, or the end of the study period (September 30, 2014).

For evaluating the association of CYP2D6 metabolizer activity with the risk of hospitalization or an ED visit, the Kaplan-Meier plots was used to visualize the relation between the metabolizer groups and outcomes. Next, Cox proportional hazard models was performed to estimate hazard ratios (HRs) and $95 \%$ confidence intervals (CIs), adjusting for both age and sex. Likelihood ratio tests with 6 degrees of freedom were applied to assess the overall significance of each of the 7 CYP2D6 phenotypes for risk of hospitalization and ED visits.

To assess the effect of disease burden on the association, we conducted analyses stratified by Minnesota tiers (tier 2 or higher vs tier 0 or 1). For this analysis, CYP2D6 metabolism phenotypes were collapsed into 3 categories (ultrarapid, poor, and the other 5 categories) for better statistical power. For each analysis (overall, lower comorbidity, and higher comorbidity), the overall significance of the 3 collapsed CYP2D6 phenotype categories was tested with likelihood ratio tests with 2 degrees of freedom. Corresponding overall $P$-values and individual HRs for CYP2D6 phenotypes were used for comparing the ultrarapid or poor category to the other 5 categories.

To indirectly assess the potential effect of medication use on the association of CYP2D6 with hospitalization or an ED visit, we calculated the prevalence of 4 disease groups (depression, arthritis, coronary artery disease, and congestive heart failure) that are enriched for use of medications metabolized with CYP2D6. The prevalence of each disease as of January 1, 2005, based on an EHR search of the preceding 5 years, was calculated for each CYP2D6 phenotype. In addition, we calculated the prevalence of common medication use.

All statistical analyses were performed with $\mathrm{R}$ statistical software (https://www.r-project.org).

\section{Results}

\section{Patients}

Of the 929 patients included in the study, the median age on January 1, 2005 was 49 years (interquartile range, $46-52$ years), and $54 \%$ were females. At the extremes of the CYP2D6 metabolism phenotype, 69 patients (7\%) were poor metabolizers and $74(8 \%)$ were ultrarapid metabolizers (Table 1). Minnesota medical tiering calculated at the month of MCB enrollment was available for 918 patients; $686(75 \%)$ were tier 0 or 1 (ie, they had $\leq 3$ comorbid health conditions). There were no notable associations of CYP2D6 phenotype with age $(P=0.46)$ or sex $(P=0.08)$ (Table 1$)$, but patients with the ultrarapid or extensive-to-ultrarapid metabolizer phenotype had a higher comorbidity score (Minnesota 
Table I Characteristics of patients by CYP2D6 metabolizer phenotype

\begin{tabular}{|c|c|c|c|c|c|c|c|c|c|}
\hline \multicolumn{10}{|l|}{ CYP2D6 phenotypes } \\
\hline Feature & $\begin{array}{l}\text { Overall } \\
\text { cohort }\end{array}$ & Ultrarapid & $\begin{array}{l}\text { Extensive } \\
\text { to } \\
\text { ultrarapid }\end{array}$ & Extensive & $\begin{array}{l}\text { Intermediate } \\
\text { to extensive }\end{array}$ & Intermediate & $\begin{array}{l}\text { Poor to } \\
\text { intermediate }\end{array}$ & Poor & $P$-value \\
\hline Patients, n (\%) & $929(100)$ & $74(8.0)$ & $145(16)$ & $195(2 \mathrm{I})$ & $179(19)$ & $203(22)$ & $64(6.9)$ & $69(7.4)$ & - \\
\hline $\begin{array}{l}\text { Age, median (IQR), } \\
\text { years }\end{array}$ & $49(46-52)$ & $49(45-52)$ & $48(47-52)$ & $48(45-52)$ & $49(46-5 I)$ & $49(45-52)$ & $49(47-5 I)$ & $50(46-52)$ & 0.46 \\
\hline Female, $\%$ & 54 & 62 & 50 & 53 & 49 & 62 & 53 & 49 & 0.08 \\
\hline Minnesota tier, \% & & & & & & & & & 0.03 \\
\hline 0 & 13 & 11 & 5.6 & 13 & 18 & 17 & 7.8 & 8.8 & \\
\hline I & 62 & 53 & 65 & 64 & 56 & 63 & 73 & 65 & \\
\hline 2 & 20 & 27 & 26 & 19 & 19 & 15 & 14 & 22 & \\
\hline 3 & 4.9 & 6.8 & 2.8 & 4.7 & 6.2 & 5.0 & 4.7 & 4.4 & \\
\hline 4 & 0.5 & 2.7 & 6.9 & 0 & 0.6 & 0.5 & 0 & 0 & \\
\hline \multicolumn{10}{|l|}{ Disease, ${ }^{\mathrm{a}} \%$} \\
\hline Depression & 7.0 & 9.4 & 8.9 & 5.8 & 6.1 & 6.9 & 2.7 & 10.5 & 0.44 \\
\hline Arthritis & 5.7 & 10.6 & 5.7 & 4.8 & 5.1 & 6.5 & 5.4 & 2.6 & 0.45 \\
\hline $\begin{array}{l}\text { Coronary artery } \\
\text { disease }\end{array}$ & 1.2 & 1.2 & 1.3 & 1.4 & 1.5 & 1.4 & 0 & 0 & 0.90 \\
\hline $\begin{array}{l}\text { Congestive heart } \\
\text { failure }\end{array}$ & 0 & 0 & 0 & 0 & 0 & 0 & 0 & 0 & - \\
\hline Smoking status, ${ }^{\mathrm{b}} \%$ & & & & & & & & & 0.72 \\
\hline Current & 3.6 & 5.9 & 2.6 & 3.9 & 2.1 & 3.3 & 5.4 & 5.3 & \\
\hline Former smoker & 28.3 & 31.8 & 28.2 & 31.9 & 28.7 & 26.5 & 25.7 & 21.3 & \\
\hline Never & 68.1 & 62.4 & 69.2 & 64.3 & 69.2 & 70.2 & 68.9 & 73.3 & \\
\hline Alcohol & & & & & & & & & 0.28 \\
\hline \multicolumn{10}{|l|}{ consumption, ${ }^{\mathrm{b}} \%$} \\
\hline Never & 14.5 & 16.5 & 10.3 & 12.1 & 19.1 & 15.9 & 17.6 & 9.2 & \\
\hline Once monthly or less & 22.3 & 22.4 & 19.9 & 24.2 & 20.1 & 21.5 & 32.4 & 19.7 & \\
\hline 2-4 times monthly & 27.7 & 24.7 & 32.7 & 29.5 & 25.8 & 26.2 & 21.6 & 31.6 & \\
\hline 2-5 times weekly & 27.5 & 30.6 & 23.7 & 28.0 & 28.4 & 29.4 & 20.3 & 30.3 & \\
\hline$\geq 6$ times weekly & 8.0 & 5.9 & 13.5 & 6.3 & 6.7 & 7.0 & 8.1 & 9.2 & \\
\hline \multicolumn{10}{|l|}{ Medication use, c \% } \\
\hline Oxycodone & 53.3 & 63.5 & 54.4 & 52.4 & 50.0 & 54.6 & 47.3 & 52.6 & 0.44 \\
\hline Codeine & 38.9 & 43.5 & 34.2 & 39.9 & 38.3 & 38.9 & 48.6 & 32.9 & 0.37 \\
\hline Hydrocodone & 35.9 & 45.9 & 39.2 & 34.1 & 33.7 & 32.9 & 32.4 & 40.8 & 0.30 \\
\hline Tramadol & 26.4 & 28.2 & 27.2 & 23.1 & 24.0 & 30.6 & 23.0 & 28.9 & 0.60 \\
\hline Morphine & 10.9 & 22.4 & 9.5 & 10.6 & 11.7 & 10.2 & 8.1 & 3.9 & 0.01 \\
\hline Metoprolol & 10.5 & 11.8 & 13.3 & II.I & 8.2 & 9.7 & 9.5 & 10.5 & 0.82 \\
\hline Amitriptyline & 6.2 & 9.4 & 10.1 & 3.4 & 3.6 & 7.4 & 5.4 & 6.6 & 0.07 \\
\hline
\end{tabular}


the prevalence date. ${ }^{b}$ Frequency data were collected upon enrollment in the Mayo Clinic Biobank. ${ }^{\circ}$ The proportion for each medication was calculated from the medication prescription history ( $\geq \mathrm{I}$ prescription for each medication) in the electronic health record.

Abbreviations: CYP2D6, cytochrome P450 2D6; IQR, interquartile range.

tier $\geq 2$ ) compared to the other phenotype groups (Table 1). Frequencies of health-related behaviors such as smoking status and alcohol consumption were similar across the 7 metabolism phenotypes ( $P=0.72$ for smoking status; $P=0.28$ for alcohol consumption). Among the 4 diseases considered, depression (7\%) and arthritis (5.7\%) were most prevalent and heart diseases were uncommon $(1.2 \%$ for coronary artery disease; $0 \%$ for congestive heart failure). Of the 7 commonly used medications (ie, used by $\geq 5 \%$ of patients) among those related to potential adverse events or inadequate efficacy with poor or ultrarapid metabolism of CYP2D6, the most commonly used were oxycodone (53\%), codeine
(39\%), and hydrocodone (36\%). Frequency of use of these 3 medications was similar across the 7 metabolism phenotypes ( $P=0.44, P=0.37$, and $P=0.30$, respectively). Morphine use was highest among ultrarapid metabolizers compared to other metabolism categories (eg, 22\% among ultrarapid vs $11 \%$ among extensive metabolizers).

\section{Hospitalization and ED visits}

In the cohort of 929 patients, 285 had at least 1 hospitalization and 496 had at least 1 ED visit from January 1, 2005 through September 30, 2014 (Table 2). With the CYP2D6 extensive metabolizers used as the reference group, the risk 
of hospitalization or an ED visit was higher for the ultrarapid metabolizers (Figures 1 and 2A, B; Table 2): 47\% of ultrarapid metabolizers were hospitalized compared to $30 \%$ of extensive metabolizers during follow-up (HR, 1.69; 95\% CI, 1.11-2.57). There was no association for risk of hospitalization between patients with the poor CYP2D6 metabolism phenotype and those with the extensive phenotype (Table 2). The risk of an ED visit was higher for the ultrarapid metabolizers (62\% sought care in the ED) compared to the extensive metabolizers (49\% visited the ED) (HR, 1.50; 95\% CI,

Table 2 Association between CYP2D6 metabolizer phenotype and the risk of hospitalization or an ED visit for 929 adults $^{\mathrm{a}}$

\begin{tabular}{|c|c|c|c|c|}
\hline \multirow{2}{*}{$\begin{array}{l}\text { CYP2D6 } \\
\text { metabolizer } \\
\text { phenotype }\end{array}$} & \multicolumn{2}{|c|}{ Hospitalization } & \multicolumn{2}{|l|}{ ED visit } \\
\hline & $\begin{array}{l}\text { Patients, } \\
\text { n (\%) }\end{array}$ & $\begin{array}{l}\text { Hazard } \\
\text { ratio } \\
(95 \% \mathrm{Cl})^{b}\end{array}$ & $\begin{array}{l}\text { Patients, } \\
\text { n (\%) }\end{array}$ & $\begin{array}{l}\text { Hazard } \\
\text { ratio } \\
(95 \% \mathrm{Cl})^{c}\end{array}$ \\
\hline Itrarapid ( $n=74)$ & $35(47)$ & $\begin{array}{l}1.69 \\
(1.11-2.57)\end{array}$ & $46(62)$ & $\begin{array}{l}1.50 \\
(1.05-2.14)\end{array}$ \\
\hline $\begin{array}{l}\text { Extensive to } \\
\text { ultrarapid }(n=145)\end{array}$ & $47(32)$ & $\begin{array}{l}1.05 \\
(0.72-1.54)\end{array}$ & $85(59)$ & $\begin{array}{l}1.35 \\
(1.00-1.80)\end{array}$ \\
\hline Extensive $(n=195)$ & $59(30)$ & Reference & $95(49)$ & Reference \\
\hline $\begin{array}{l}\text { Intermediate to } \\
\text { extensive }(n=179)\end{array}$ & $45(25)$ & $\begin{array}{l}0.78 \\
(0.53-1.16)\end{array}$ & $88(49)$ & $\begin{array}{l}1.04 \\
(0.78-1.38)\end{array}$ \\
\hline $\begin{array}{l}\text { Intermediate } \\
(\mathrm{n}=203)\end{array}$ & $58(29)$ & $\begin{array}{l}0.91 \\
(0.63-1.31)\end{array}$ & $108(53)$ & $\begin{array}{l}1.14 \\
(0.87-1.51)\end{array}$ \\
\hline $\begin{array}{l}\text { Poor to } \\
\text { intermediate }(n=64)\end{array}$ & $20(3 \mathrm{I})$ & $\begin{array}{l}1.04 \\
(0.63-1.73)\end{array}$ & $31(48)$ & $\begin{array}{l}1.02 \\
(0.68-1.53)\end{array}$ \\
\hline Poor $(n=69)$ & $21(30)$ & $\begin{array}{l}0.95 \\
(0.58-1.56)\end{array}$ & $43(62)$ & $\begin{array}{l}1.38 \\
(0.96-1.98)\end{array}$ \\
\hline
\end{tabular}

Notes: adjusted for age and sex. ${ }^{b}$ Overall $P=0.07$. ' Overall $P=0.14$

Abbreviations: $\mathrm{Cl}$, confidence interval; CYP2D6, cytochrome P450 2D6; ED, emergency department.

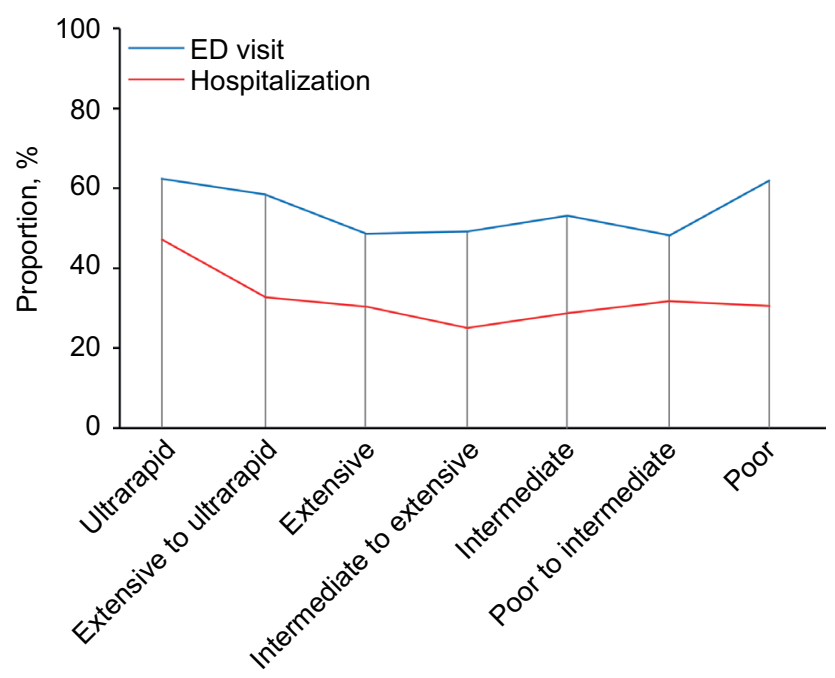

CYP2D6 phenotype

Figure I Proportion of hospitalizations and ED visits among 929 patients according to CYP2D6 phenotype.

Notes: A higher proportion of patients with the ultrarapid metabolizer phenotype were hospitalized or visited the ED, compared to patients with the extensive metabolizer phenotype.

Abbreviations: CYP2D6, cytochrome P450 2D6; ED, emergency department.
1.05-2.14). The risk of an ED visit for patients with the poor metabolizer phenotype was also higher compared to the risk for patients with the extensive phenotype (HR, 1.38; 95\% CI, 0.96-1.98), although the difference was not statistically significant.

\section{Impact of comorbidity level}

In exploratory analyses, we stratified the CYP2D6 phenotypes and the risk of hospitalization or an ED visit by the level of comorbidity, defined as Minnesota tiers 0 or 1 (lower disease burden) or 2 or higher (ie, higher disease burden) (Table 3 ). The risk of hospitalization for ultrarapid metabolizers (compared to the reference group of the 5 CYP2D6 phenotypes between ultrarapid and poor activity) was higher among participants with lower disease burden (HR, 1.85; 95\% CI, 1.14-3.00), but was attenuated for participants with higher disease burden (HR, 1.38; 95\% CI, 0.80-2.35). The risk of hospitalization for patients with the poor metabolism phenotype did not vary by the level of comorbidity.

The risk of an ED visit for ultrarapid metabolizers (compared to the 5 reference CYP2D6 phenotypes) was higher for patients with higher disease burden (HR, 1.51; 95\% CI, 0.94-2.41) compared to those with lower disease burden (HR, 1.14; 95\% CI, 0.76-1.72). Similarly, the risk of an ED visit for poor metabolizers (compared to the 5 reference CYP2D6 phenotypes) was higher among patients with higher disease burden (HR, 1.56; 95\% CI, 0.91-2.68) compared to those with lower disease burden (HR, 1.15; 95\% CI, 0.78-1.70). The interaction between disease burden (lower vs higher) and CYP2D6 metabolism phenotypes (ultrarapid, poor, and the middle 5 categories) was not significant for hospitalization $(P=0.18)$ or an $\mathrm{ED}$ visit $(P=0.44)$; the power was low for these tests.

\section{Discussion}

In this cohort study of 929 adults in a primary care practice, we found that ultrarapid metabolism of CYP2D6 was associated with a higher risk of hospitalization or an ED visit, compared to extensive metabolism. Finding potential pharmacogenomic predictors in a middle-aged population with relatively few comorbid health conditions is encouraging because most clinical models used for predicting hospitalization have been designed for older patients with multiple chronic conditions. ${ }^{12,17,18}$ In stratified analysis, we found that ultrarapid metabolism of CYP2D6 was associated with a higher risk of hospitalization compared to the extensive metabolism phenotype among stratified patients with a lower comorbidity burden. This provides a unique finding in a population that has few traditional predictors of hospitalization. 


\section{A}

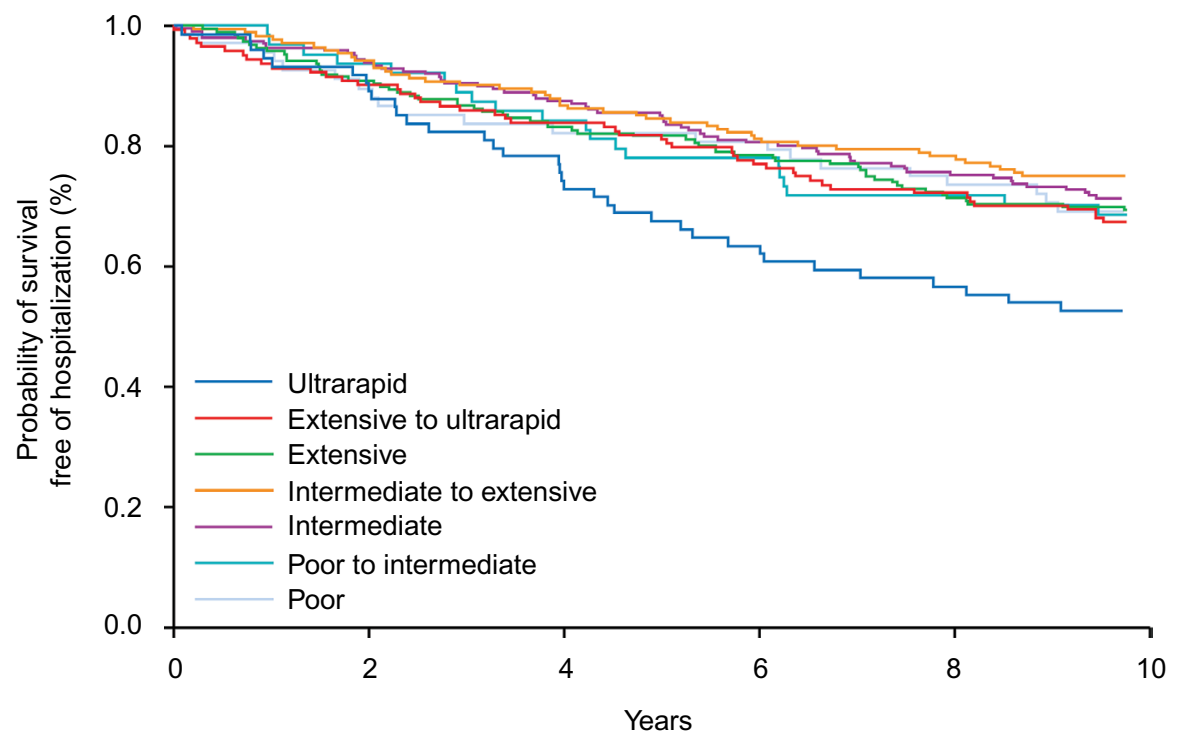

B

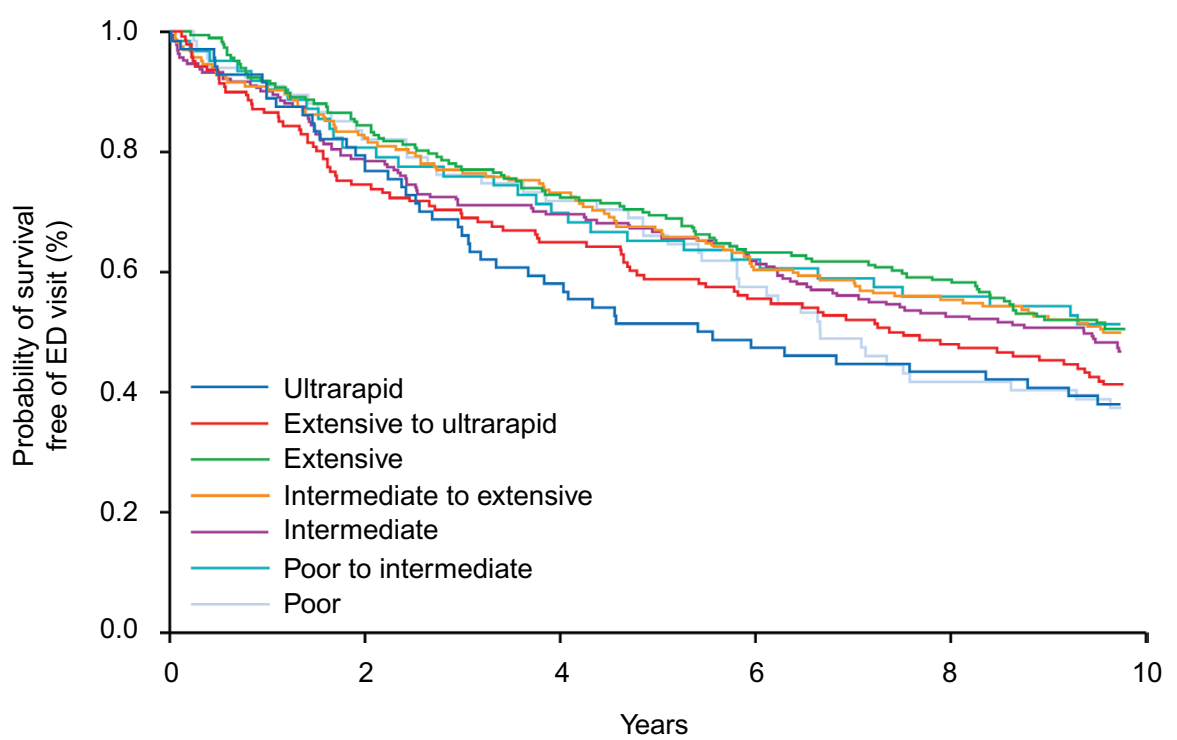

Figure 2 Survival analysis according to CYP2D6 phenotype.

Notes: (A) Probability of survival free of hospitalization according to CYP2D6 phenotype of 929 patients. Patients with the ultrarapid metabolizer phenotype had a higher rate of hospitalization compared to patients with the extensive metabolizer phenotype. (B) Probability of survival free of ED visit according to CYP2D6 phenotype of 929 patients. Patients with the ultrarapid metabolizer phenotype had a higher rate of ED use compared to patients with the extensive metabolizer phenotype.

Abbreviations: CYP2D6, cytochrome P450 2D6; ED, emergency department.

Many traditional clinical models predict hospitalization on the basis of age 17 or comorbid health conditions. ${ }^{12,13}$ Thus, pharmacogenomics provides a unique risk factor for predicting hospitalization among healthy people, as opposed to older and sicker populations. Pharmacogenomics also offers treatment options, such as those attempted in some cardiology studies. ${ }^{19}$ In a study of 149 patients admitted to the hospital for depressive disorder, the LOS was longer for patients with lower CYP2D6 activity than for those with higher activity ( 7.8 vs 5.7 days; $P=0.002) .{ }^{3}$ However, criticisms of the study include a lack of defined metabolism phenotype..$^{20}$ In a second small study of patients who had depression or schizophrenia, the median number of hospital days was higher for patients with poor CYP2D6 activity than for those with other phenotypes (median, 57.5 vs 40 days). ${ }^{4}$

In the study, we discovered that patients with the ultrarapid CYP2D6 phenotype were more likely to visit the ED, compared to those with the extensive phenotype. In a different setting, there was no difference in ED use among CYP2D6 phenotypes in 56 patients who were being treated with codeine for sickle cell disease. ${ }^{21}$ That study was small; thus, the number of ultrarapid metabolizers would also be small, 
Table 3 Association between CYP2D6 metabolizer phenotype and the risk of hospitalization or an ED visit for 929 adults, stratified by comorbidity level ${ }^{\mathrm{a}, \mathrm{b}}$

\begin{tabular}{|c|c|c|c|c|c|c|c|c|c|}
\hline \multirow[t]{2}{*}{ Outcome } & \multicolumn{3}{|c|}{ Overall cohort ( $\mathrm{N}=929)$} & \multicolumn{3}{|c|}{ Lower comorbidity $\left(n=686^{c}\right)$} & \multicolumn{3}{|c|}{ Higher comorbidity $\left(n=232^{c}\right)$} \\
\hline & $\begin{array}{l}\text { Patients, } \\
\text { n }\end{array}$ & $\begin{array}{l}\text { Events, } \\
\text { n (\%) }\end{array}$ & $\mathrm{HR}^{\mathrm{d}}(\mathbf{9 5} \% \mathrm{Cl})$ & $\begin{array}{l}\text { Patients, } \\
\text { n }\end{array}$ & $\begin{array}{l}\text { Events, } \\
\text { n (\%) }\end{array}$ & $\mathrm{HR}^{\mathrm{d}}(95 \% \mathrm{Cl})$ & $\begin{array}{l}\text { Patients, } \\
\text { n }\end{array}$ & $\begin{array}{l}\text { Events, } \\
\text { n (\%) }\end{array}$ & $\mathrm{HR}^{\mathrm{d}}(\mathbf{9 5} \% \mathrm{Cl})$ \\
\hline Hospitalization & & & $P=0.004$ & & & $P=0.03$ & & & $P=0.23$ \\
\hline Ultrarapid & 74 & $35(47)$ & $1.80(1.26-2.57)$ & 47 & $19(40)$ & $1.85(1.14-3.00)$ & 27 & $16(59)$ & $1.38(0.80-2.35)$ \\
\hline Other 5 categories $^{\mathrm{e}}$ & 786 & $229(29)$ & Reference & 589 & $139(24)$ & & 187 & $86(46)$ & Reference \\
\hline Poor & 69 & $21(30)$ & $1.09(0.86-1.38)$ & 50 & $16(32)$ & $\begin{array}{l}\text { Reference } \\
\text { I.3। (0.78-2.20) }\end{array}$ & 18 & $5(28)$ & $0.57(0.23-1.40)$ \\
\hline ED visit & & & $P=0.08$ & & & $P=0.67$ & & & $P=0.10$ \\
\hline Ultrarapid & 74 & $46(62)$ & $1.35(1.00-1.84)$ & 47 & $25(53)$ & $1.14(0.76-1.72)$ & 27 & $21(78)$ & $I .5 I(0.94-2.4 I)$ \\
\hline Other 5 categories ${ }^{e}$ & 786 & $407(52)$ & Reference & 589 & $290(49)$ & Reference & 187 & $113(60)$ & Reference \\
\hline Poor & 69 & $43(62)$ & I.24 (0.9I-I.7I) & 50 & $28(56)$ & I.IS (0.78-I.70) & 18 & $15(83)$ & $1.56(0.9 \mid-2.68)$ \\
\hline
\end{tabular}

Notes: ${ }^{a}$ Comorbidity levels were based on Minnesota tiers (higher $\geq 2$; lower 0 or I). ${ }^{b}$ All analyses were adjusted for age and sex. ${ }^{\mathrm{C} C o m o r b i d i t y ~ l e v e l s ~ w e r e ~ a v a i l a b l e ~ f o r ~} 918$ patients. ${ }^{\mathrm{H}} \mathrm{Hazard}$ ratio compared ultrarapid metabolizer to the other 5 phenotypes. ${ }^{\mathrm{e}}$ The other 5 categories were I) extensive to ultrarapid, 2) extensive, 3 ) intermediate to extensive, 4) intermediate, and 5) poor to intermediate.

Abbreviations: $\mathrm{Cl}$, confidence interval; CYP2D6, cytochrome P450 2D6; ED, emergency department; HR, hazard ratio.

and the study team primarily compared poor metabolizers with no serum morphine levels and normal metabolizers with detectable morphine levels after codeine administration. There are no directly comparable studies of CYP2D6 phenotype and risk of an ED visit. Our findings for both hospitalization and ED visits require replication in other studies.

There is some biologic plausibility for increased risk of hospitalization or an ED visit for patients with the ultrarapid metabolism phenotype. For example, in patients with ultrarapid metabolism, the increased metabolism may decrease the plasma levels of the antidepressant fluoxetine, a selective serotonin reuptake inhibitor. ${ }^{22}$ Subsequently, changes in blood levels of antidepressants may have clinical effects. Potentially supporting this theory is a study of 587 patients, in which the metabolic activity of CYP2D6 and cytochrome P450 2C19 was higher in patients who attempted suicide than in patients who did not attempt suicide (odds ratio, 1.37 ; 95\% CI, $1.05-1.78 ; P=0.02) .{ }^{23}$ With potential variation in the efficacy of antidepressants, some authors have recommended dose changes based on CYP2D6 activity. ${ }^{24}$ However, the potential effect of ultrarapid metabolism on other drugs besides antidepressants (which are metabolized by CYP2D6) is less clear. For example, use of metoprolol, a common $\beta$-blocker for heart disease, did not appear to show a relationship between titration of metoprolol according to an understanding of the polymorphisms and episodes of congestive heart failure. ${ }^{25}$

The present study has limitations. If patients were hospitalized somewhere besides Mayo Clinic, the result would be missing outcome data. However, it is unlikely that patients would differentially be hospitalized elsewhere according to their CYP2D6 phenotype; thus, a systematic bias should be minimized. The patients in the RIGHT study were predominantly European Americans ${ }^{11}$; thus, these findings may not apply to other populations. However, the results from Olmsted County, Minnesota, and surrounding counties may apply to the Upper Midwest. ${ }^{26}$ We were able to report diseases that account for the common uses of CYP2D6 medications and for any use by drug class. However, we could not analyze specific medications because of the low prevalence of use of individual medications and the practical difficulties in capturing the specifics of medication use in the relevant time frame before hospitalization or an ED visit. We also did not directly measure drug-gene interaction in this study. Thus, we cannot ascribe the association of the CYP2D6 phenotype and hospitalization to any specific medication. Although classifying or grouping medications into categories could increase power, it may not reflect the effect of individual medications. However, multiple drugs (prescribed and over the counter), polypharmacy (particularly in older patients), and other environmental (nonmedication) exposures (eg, smoking, caffeine, marijuana, and workplace chemicals) are likely to interact in multiple and complex ways with the CYP2D6 phenotype that could not be predicted from the study of single medications. ${ }^{27}$ Thus, the CYP2D6 phenotype may serve as a better summary risk marker of the risk of hospitalization and an ED visit than individual exposures; this hypothesis needs further testing.

\section{Conclusion}

The application of pharmacogenomics to precision medicine is an important step in translating genomic medicine to clinical practice. In the study, ultrarapid CYP2D6 metabolizer status was associated with increased risk of hospitalization and ED visits, when compared to extensive metabolizer 
status. Thus, there may be clinical utility in preemptively genotyping patients to decrease health care use. Of importance, this predicted hospitalization occurred among middleaged patients who were mostly healthy. This study helps to build the argument for pharmacogenomic testing for health care groups and health plans, although the clinical utility and cost-effectiveness will need to be evaluated more rigorously. This would require investment in the bioinformatics and clinical informatics infrastructure to support pharmacogenomics in clinical practice. Applications of pharmacogenomics can augment clinical risk predictors and provide personalized medicine for patients.

\section{Acknowledgments}

We acknowledge Kari Takahashi, MEd, for copyediting the manuscript.

This work was supported in part by Mayo Clinic Center for Individualized Medicine, Robert D. and Patricia E. Kern Center for the Science of Health Care Delivery, National Institutes of Health grants U19 GM61388 (The Pharmacogenomics Research Network), R01 GM28157, U01 HG005137, R01 CA138461, R01 AG034676 (The Rochester Epidemiology Project), and U01 HG06379 and U01 HG06379 Supplement (The Electronic Medical Record and Genomics [eMERGE] Network).

\section{Disclosure}

PYT serves on the medical advisory board for Axial, LLC. JLB and Mayo Clinic have licensed pharmacogenomic drug selection algorithms to Assurex Health and OneOme. The other authors report no conflicts of interest in this work.

\section{References}

1. Mannesse CK, Derkx FH, de Ridder MA, Man in 't Veld AJ, van der Cammen TJ. Contribution of adverse drug reactions to hospital admission of older patients. Age Ageing. 2000;29(1):35-39.

2. Zanger UM, Schwab M. Cytochrome P450 enzymes in drug metabolism: regulation of gene expression, enzyme activities, and impact of genetic variation. Pharmacol Ther. 2013;138(1):103-141.

3. Ruano G, Szarek BL, Villagra D, et al. Length of psychiatric hospitalization is correlated with CYP2D6 functional status in inpatients with major depressive disorder. Biomark Med. 2013;7(3): 429-439.

4. Kropp S, Lichtinghagen R, Winterstein K, Schlimme J, Schneider U. Cytochrome P-450 2D6 and 2C19 polymorphisms and length of hospitalization in psychiatry. Clin Lab. 2006;52(5-6):237-240.

5. Yep T, Devine B. A cost utility analysis of CYP2D6 pharmacogenetic guided dosing versus standard dosing of risperidone for treatment of schizophrenia. Value Health. 2014;17(3):A217.

6. Kelly LE, Rieder M, van den Anker J, et al. More codeine fatalities after tonsillectomy in North American children. Pediatrics. 2012;129(5): e1343-e1347.

7. Rieder MJ, Carleton B. Pharmacogenomics and adverse drug reactions in children. Front Genet. 2014;5:78.
8. Samer CF, Lorenzini KI, Rollason V, Daali Y, Desmeules JA. Applications of CYP450 testing in the clinical setting. Mol Diagn Ther. 2013;17(3): $165-184$.

9. Goetz MP, Knox SK, Suman VJ, et al. The impact of cytochrome P450 2D6 metabolism in women receiving adjuvant tamoxifen. Breast $\mathrm{Cancer}$ Res Treat. 2007;101(1):113-121.

10. Olson JE, Ryu E, Johnson KJ, et al. The Mayo Clinic Biobank: a building block for individualized medicine. Mayo Clin Proc. 2013;88(9): 952-962.

11. Bielinski SJ, Olson JE, Pathak J, et al. Preemptive genotyping for personalized medicine: design of the right drug, right dose, right timeusing genomic data to individualize treatment protocol. Mayo Clin Proc. 2014;89(1):25-33.

12. Haas LR, Takahashi PY, Shah ND, et al. Risk-stratification methods for identifying patients for care coordination. Am J Manag Care. 2013; 19(9):725-732.

13. Weiner JP, Starfield BH, Steinwachs DM, Mumford LM. Development and application of a population-oriented measure of ambulatory care case-mix. Med Care. 1991;29(5):452-472.

14. Black JL 3rd, Walker DL, O'Kane DJ, Harmandayan M. Frequency of undetected CYP2D6 hybrid genes in clinical samples: impact on phenotype prediction. Drug Metab Dispos. 2012;40(1):111-119.

15. Kramer WE, Walker DL, O'Kane DJ, et al. CYP2D6: novel genomic structures and alleles. Pharmacogenet Genomics. 2009;19(10):813-822.

16. Ji Y, Skierka JM, Blommel JH, et al. Preemptive pharmacogenomic testing for precision medicine: a comprehensive analysis of five actionable pharmacogenomic genes using next-generation DNA sequencing and a customized CYP2D6 genotyping cascade. J Mol Diagn. 2016;18(3):438-445.

17. Crane SJ, Tung EE, Hanson GJ, Cha S, Chaudhry R, Takahashi PY. Use of an electronic administrative database to identify older community dwelling adults at high-risk for hospitalization or emergency department visits: the elders risk assessment index. BMC Health Serv Res. 2010;10:338.

18. Naessens JM, Stroebel RJ, Finnie DM, et al. Effect of multiple chronic conditions among working-age adults. Am J Manag Care. 2011;17(2):118-122.

19. Khalighi B, Wu Y, Fan WQ, et al. CYP2D6 genetic information-guided metoprolol use in a cardiology clinic: a perspective study. Cardiology. 2014;128(Suppl 1):159.

20. Penas-Lledo EM, Llerena A. CYP2D6 genetic polymorphism and psychiatry patients' hospitalization period. Biomark Med. 2013; 7(6):915-916.

21. Shord SS, Cavallari LH, Gao W, et al. The pharmacokinetics of codeine and its metabolites in blacks with sickle cell disease. Eur J Clin Pharmacol. 2009;65(7):651-658.

22. Hamelin BA, Turgeon J, Vallee F, Belanger PM, Paquet F, LeBel M. The disposition of fluoxetine but not sertraline is altered in poor metabolizers of debrisoquin. Clin Pharmacol Ther. 1996;60(5):512-521.

23. Penas-Lledo E, Guillaume S, Naranjo ME, et al. A combined high CYP2D6-CYP2C19 metabolic capacity is associated with the severity of suicide attempt as measured by objective circumstances. Pharmacogenomics J. 2015;15(2):172-176.

24. Kirchheiner J, Brosen K, Dahl ML, et al. CYP2D6 and CYP2C19 genotype-based dose recommendations for antidepressants: a first step towards subpopulation-specific dosages. Acta Psychiatr Scand. 2001;104(3):173-192.

25. Terra SG, Pauly DF, Lee CR, et al. Beta-adrenergic receptor polymorphisms and responses during titration of metoprolol controlled release/extended release in heart failure. Clin Pharmacol Ther. 2005; 77(3):127-137.

26. St Sauver JL, Grossardt BR, Leibson CL, Yawn BP, Melton LJ 3rd, Rocca WA. Generalizability of epidemiological findings and public health decisions: an illustration from the Rochester Epidemiology Project. Mayo Clin Proc. 2012;87(2):151-160.

27. Heim MH, Meyer UA. Evolution of a highly polymorphic human cytochrome P450 gene cluster: CYP2D6. Genomics. 1992;14(1):49-58. 


\section{Publish your work in this journal}

Pharmacogenomics and Personalized Medicine is an international, peerreviewed, open access journal characterizing the influence of genotype on pharmacology leading to the development of personalized treatmen programs and individualized drug selection for improved safety, efficacy and sustainability. This journal is indexed on the American Chemical
Society's Chemical Abstracts Service (CAS). The manuscript management system is completely online and includes a very quick and fair peer-review system, which is all easy to use. Visit http://www.dovepress. com/testimonials.php to read real quotes from published authors.

Submit your manuscript here: https://www.dovepress.com/pharmacogenomics-and-personalized-medicine-journal 